\title{
Implementation of the Tree Counting Process in the Process of Urban Reclamation with the use of Fuzzy Neural Network for Agro forestry
}

\author{
Sergey E. Lazarev ${ }^{1}$, Alexandra V. Semenyutina ${ }^{2}$, Alexander I. Belyaev ${ }^{3}$ \\ ${ }^{1}$ Federal scientific center of agro-ecology, integrated land reclamation and protective afforestation of \\ the Russian Academy of Sciences, Volgograd, Russia, Hortus@yandex.ru \\ ${ }^{2}$ Federal scientific center of agro-ecology, integrated land reclamation and protective afforestation of \\ the Russian Academy of Sciences, Volgograd, Russia, doksemenutina@mail.ru \\ ${ }^{3}$ Federal scientific center of agro-ecology, integrated land reclamation and protective afforestation of \\ the Russian Academy of Sciences, Volgograd, Russia, info@vfanc.ru
}

\section{ABSTRACT}

Representatives of the genus Robinia are of undoubted interest for landscape architecture and garden engineering. This group of plants has high decorative properties and has sufficient polymorphism to create homogeneous landscape compositions.

As a result of the research, promising species were identified for creating woodlands (R. pseudoacacia), groves (R. pseudoacacia x R. neomexicana, R. neomexicana var. rusbyi, R. neomexicana var. neomexicana), alleys (R. pseudoacacia f. pyramidalis, R. pseudoacacia f.umbraculifera), tapeworms (R. viscosa var. hartwegii).

The types, varieties and forms that are promising for group plantings are determined. Based on taxational and phenological studies, optimal combinations of different representatives of the genus Robinia were determined. To develop a range odnomodovykh wood developed a decorative scheme compatibility and placement of the genus Robinia, given the color of flowers, length of decorative flowering period and plant height. The technology is used for counting trees MIMO. Directions improvement WiMAX systems with MIMO depend on select a method adaptations K systems changing ones characteristics wireless channel, and this depends on availability or omissions line of sight. Depending on this task adaptations resolved by-different. Behind available direct line of sight increases dramatically degree of correlation signals in MIMO channels that reduces efficiency MIMO. In this case needand or significant spatial resolution posting antennas, or organization customer relations on a narrow road ray that generated accordingly before the request. In algorithm qualities formation a bunch of narrow ones they can be used software applications methods or algorithms adaptive ones antenna arrays, synthesized data for non-stationary environment, that is, physical or mathematical formation beams. The first one physical method depends on changes in direction transfers and reception, and the second selects the best one the channel component mathematically.
The last one counts more appropriate for WiMAX stations using a smaller one complexity implementations.

Key words : Engineering, gardening, methods of embittering, types of green spaces, decorative wood groups.

\section{INTRODUCTION}

In absence conditions direct line of sight MIMO channels are provided as follows practically uncorrelated, which significantly increases the efficiency application MIMO. In MIMO systems for splitting channels are applied spatio-temporal analysis or spatio-frequency encoding. An important direction system development with MIMO is their adaptation to change parameters the channel that provides more complete information implementation available spatial data and frequency control the resource [1-4]. However, question of adaptation WiMAX systems from MIMO to real-world conditions the connections have been investigated not enough [5]. Future systems with multiple options the antennas must be connected use all resources both temporary and temporary and frequency ouch are a hey, to minimize power transfers and, in at the same time, increase pass rate ability communication systems through adaptation up to the real world quickly with status of the radio channel [6].

Like this keep it up-to-date there is a scientific problem, which consists of in development increase methods transmission quality information in systems radio access WiMAX based on usage adaptive modulations signals in spatial features MIMO channels [7].

\section{MATERIALS AND METHODS}

Mathematical description modeling processes on the physical level in the system WIMAX impossible without knowledge of the properties and the main parameters multi-beam broadband the radio channel. A model that is fully enough describes such a method channel $b$ discrete moments of time, there is a delay line with taps and a characteristic [8-11]. 
In this model parameters channels are changing to discrete ones moments of time. Usually it is considered that what is a measurement parameters what is happening with frequency $f_{s}=1 / T_{s}$, where $T_{s}$-duration the symbol. Allowing, what a time $T_{\mathrm{s}}$ parameters channels remain unchanged, output sequence data can be submitted by in the form of:

$h[t]=\left[h_{0}(t) h_{l}(t) h \ldots v(t)\right]^{T},(1)$

which one reflects basic parameters multi-beam channels into discrete ones moments of time. These parameters are: losses on distribution route; shading; fading signals associated with mipramine interference; distribution delays and a band of coherence. In the work carried out impact analysis changes to the specified parameters on the quality of communication and features usage in technology WiMAX spatial search methods and frequency control post, as well as spatio-temporal and spatial-frequency analysis encoding for reducing this negative influences. Conducted the analysis allowed make a conclusion, what's important direction system development WiMAX with MIMO is their adaptation to change parameters multi-beam a radio channel. which provides the most complete implementation available spatial data and frequency control the resource [1-5].

When creating decorative compositions, the overall size of plants and the structure of the crown are important. The largest size in the generic complex is reached by typical representatives of $\mathrm{R}$. pseudoacacia and its form, with the exception of R. pseudoacacia f. umbraculifera (DC) Rehd., whose crown rarely reaches a height of 5 meters. The height of typical representatives of R. pseudoacacia, depending on the hydrological regime in arid conditions, can vary from 8 to 11 meters at the age of 20 years. Depending on the conditions, the height of R. viscosa can reach from 5 to 10 meters. Height $\mathrm{R}$ neomexicana and its color forms at 20 years of age, depending on the conditions can also vary from 5 to 8 meters. Hybrid forms (R. pseudoacacia $x$ R. neomexicana) in similar conditions reach a height of 8-9 meters [6].

Decorative properties, taxational indicators and bioecological features in the conditions of cultivation determine the degree of prospects for using representatives of the genus Robinia in various gardening techniques (arrays, groves, curtinas, groups, alleys, tapeworms) and types of green spaces [14].

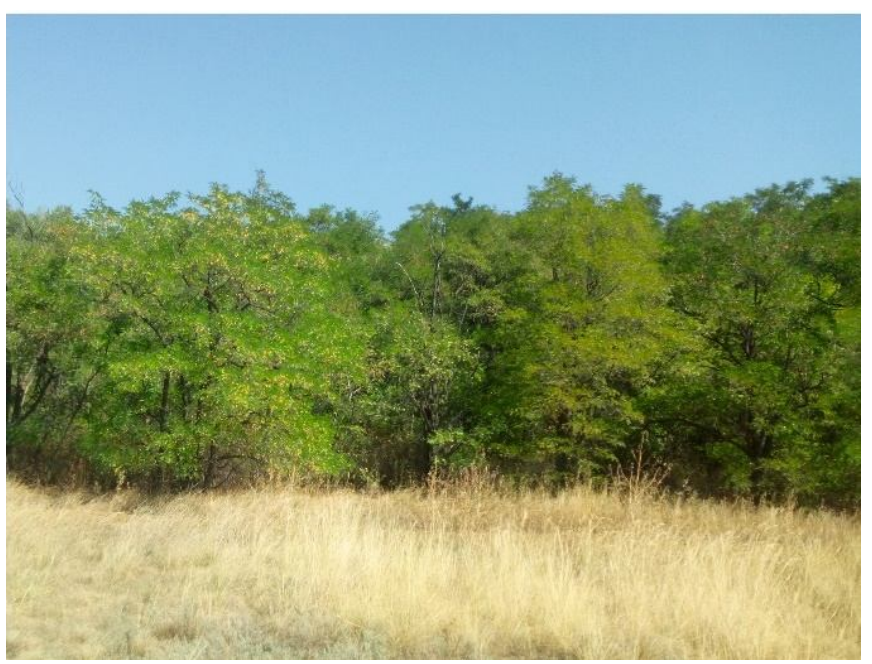

Figure 1: Tree array R. pseudoacacia
To create woodlands, sustainable species with high generative potential and a sufficient number of mother plantations for harvesting seed material are of great value. These species undoubtedly include R. pseudoacacia (figure 1).

To create small woodlands (up to $1 \mathrm{ha}$ ) or groves with a sufficient number of mother seed plantations, it is also possible to use hybrid forms of $\mathrm{R}$. pseudoacacia $\mathrm{x} R$. neomexicana with high generative potential, as well as $\mathrm{R}$. neomexicana var. rusbyi and $R$. neomexicana var. neomexicana with an average seed productivity [7] (figure 2).

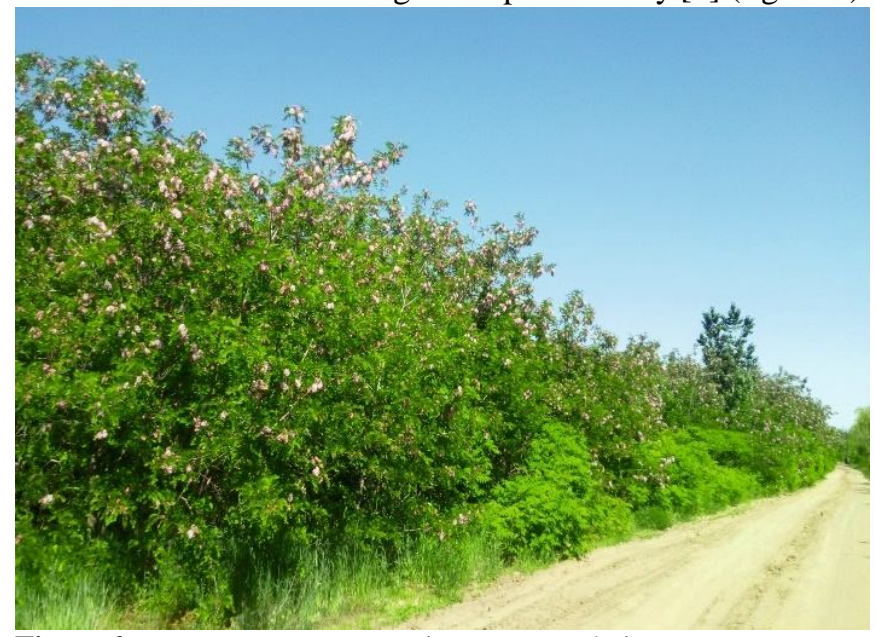

Figure 2: Tree grove R. neomexicana var. rusbyi

When creating mixed wood groups or curtins from various species and forms of the genus Robinia, it is necessary to take into account the color of the flowers, the duration of the decorative period and the overall size of the plants. So, for the background, you need to use a relatively tall R. pseudoacacia, and for the front - different types, varieties and forms of pink-flowered species. R. pseudoacacia is well combined in joint plantings with $R$. neomexicana var. rusbyi and $R$. neomexicana var.neomexicana (figure 3 ).

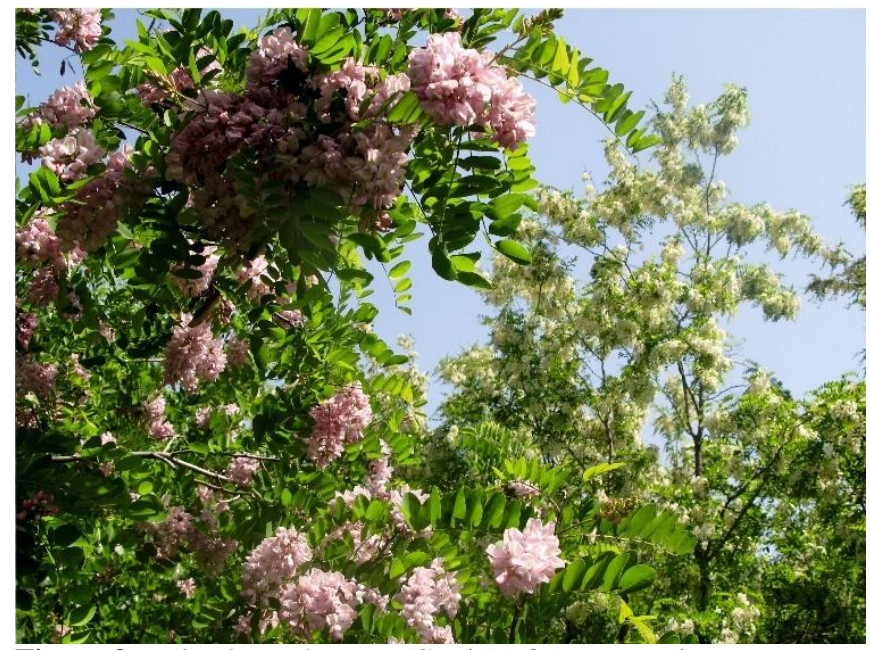

Figure 3.: Mixed wood group (Curtin) of R. neomexicana var. rusbyi and R. pseudoacacia

The light pink and pale purple forms of R. neomexicana are of interest for creating coloristic compositions in mixed wood curtins involving different types of woody plants [12]. Given the relatively late flowering period (may-June), and the small 
size of plants $(5-8 \mathrm{~m})$, these forms should be used in the foreground or to create edges in front of woodlands, groves or kurtinami with the participation of trees of the first and second magnitude. Long flowering period of $\mathrm{R}$. viscosa var. hartwegii (Koehne) Ashe and its relatively small size make it an indispensable foreground plant in mixed groups with $\mathrm{R}$. pseudoacacia, R. pseudoacacia $x$ R. neomexicana, $R$. neomexicana f. light pink, R. neomexicana f. pale purple (figure 4).

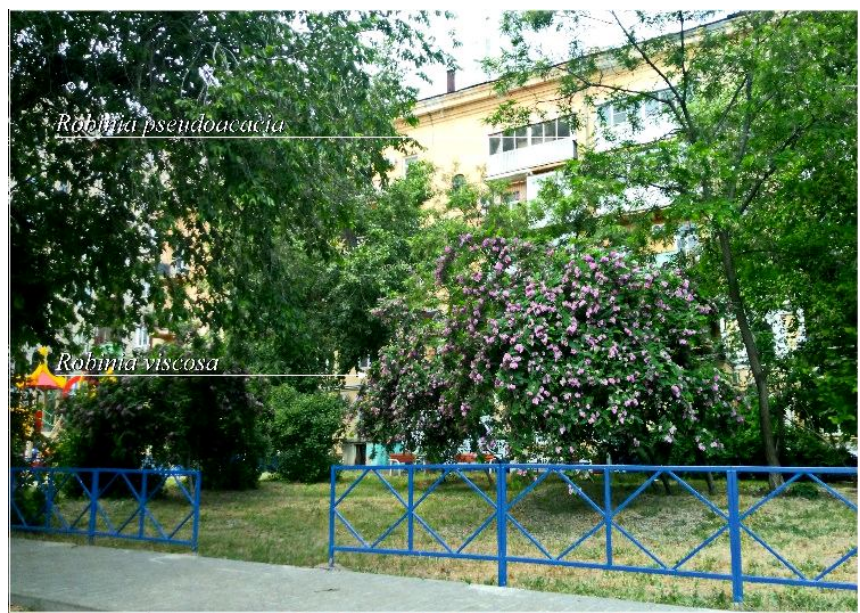

Figure 4: Mixed wood group R. viscosa var. hartwegii (Koehne) Ashe and R. Pseudoacacia

Species and intraspecific diversity of the genus Robinia allows you to create monosads and spectacular tree groups and curtins, from plants belonging to the same Botanical genus (species groups). The selection of plants for such groups should also be carried out taking into account the basic principles (plant height, duration of the decorative period and color of flowers).

To develop a range wood group proposed to use the decorative scheme compatibility and placement of the genus Robinia, given the color of flowers, length of decorative flowering period and plant height (figure 5).

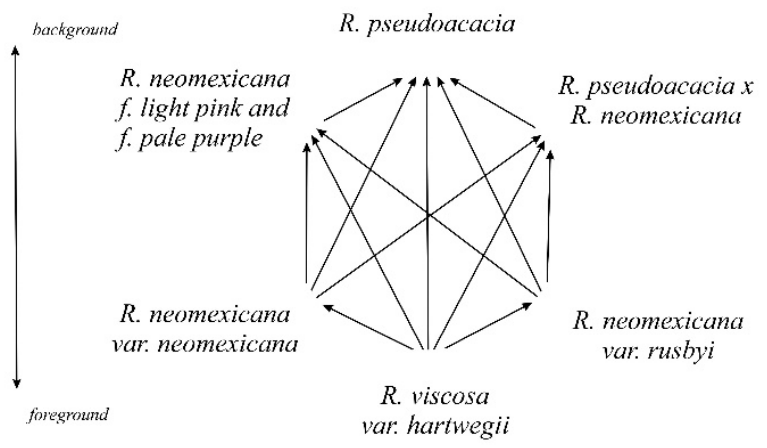

Figure 5: Scheme of decorative compatibility and placement of representatives of the genus Robinia, taking into account the color of flowers, the duration of the decorative flowering period and plant heights

This scheme allows you to select plants based on the main angle of the landscape composition. The direction of the lines in the diagram determines the location of the plants in the background and foreground [13].
The diagram clearly illustrates the decorative compatibility of representatives of the genus Robinia. The absence of lines on the diagram indicates that there is no decorative compatibility between plants. In addition, the plants located next to each other in this diagram have a relatively low degree of decorative compatibility. The highest decorative effect is observed when the plants located on the diagram are used together through a position, or opposite each other.

The system WiMAX with MIMO should use all available time and frequency bands resources to use minimize it transfers and, in at the same time, zoom in pass rate ability communication systems by adapting it up to the radio channel status. Solving this problem issues cannot be resolved without carrying out a wide range research, related to by modeling processes in the WiMAX systems with MIMO [12].

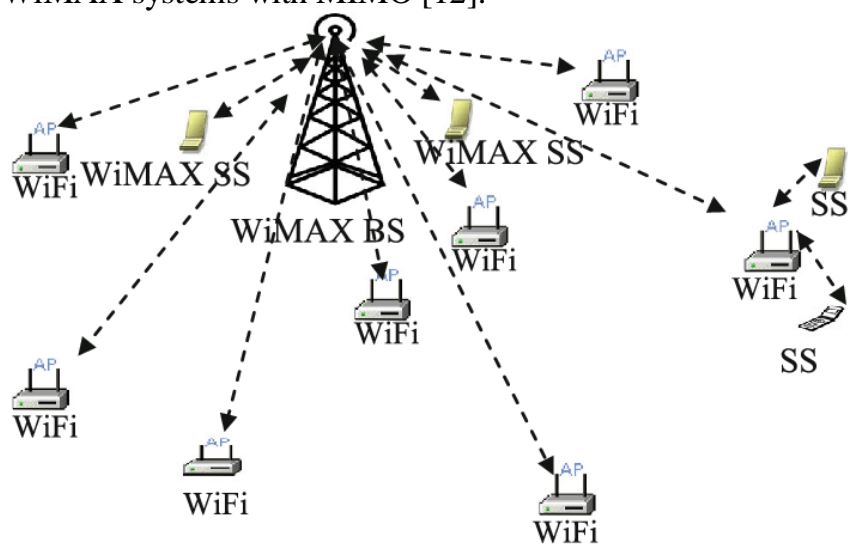

Figure 6: WiMAX network architecture

Figure 6 shows us the WiMAX network architecture for solving our problem.

OFDM modulation applied in technology WiMAX to eliminate massingale interference. This is provided by parallel sending data on different subcarriers and elongation passed characters so, so that they are much longer the largest time delay in the channel. However, at high speed transfers from using the following parameters: broadband users frequency bands channels are possible deep enough selective ads fading. In the standard WiMAX with OFDM is not expected management capacity for various purposes subcarriers and in the case of occurrence of frequency-selective fading inevitably, the deterioration is inevitable communication quality. It is obvious that and in this case usage adaptive management parameters signals in frequency band regions (reallocation subcarriers including real ones characteristics channel) acquires very important value [13].

Usage measured values element values channel matrices not completely solves the problem definition real state channel through "freeze" matrices for time packet transfers characters. For mobile devices subscribers channel matrix $\boldsymbol{N}$ can significantly in time's. This leads to towards growth errors in the definition matrices and, accordingly, to increase bit errors at reception.

In works [5,9] suggested use measured by passed test cases signals matrix value channel and enter predictions next steps element values matrices. And, because the process coefficient changes transfers to radio channel MIMO is close to Markovsky, then for 
the prediction you can use linear algorithms. During transmission useful characters information value of elements matrices are approximated by linear parameters by law from the last one measured $\mathrm{K}$ value provided for. Obviously, than less error predictions, the lower the level errors when reception. Level it depends on the number of errors depending on a number of factors: from an error measurements and errors predictions matrices and approximations functions on the site the interval between pilot projects signals and from the size interval. Reducing the level software errors reduction account the interval between pilot projects characters there is in effective because of the growth loss of bandwidth abilities systems. In offered specific method weight of test items signals in in General the amount of information passed information it is $1-2 \%$, what results to non-essential bandwidth loss software capabilities useful the signal. For reducing the number of users errors that occur when changing the matrix channel sequence counts of each the coefficient channel transfers between a pair of antennas maybe submitted by as a linear function combination their previous ones values. Like this prediction algorithm it's called linear and founded on use autoregressive ads models.

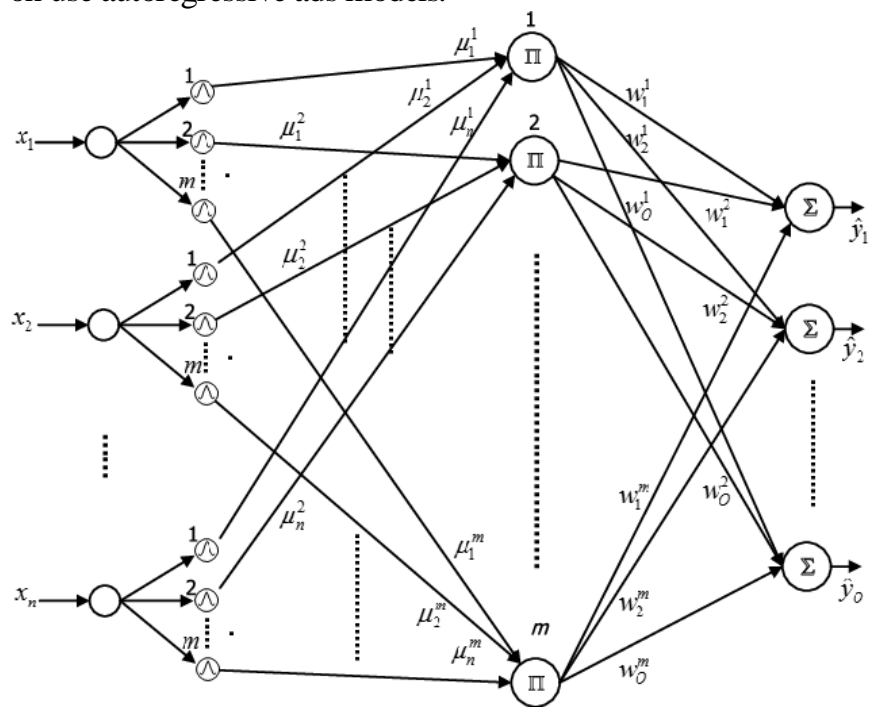

Figure 7: Fuzzy neural network architecture MIMO

Figure 7 shows us fuzzy neural network in WiMAX network. Continuity technologies WiMAX with MIMOS technical and software solution of the standard 802.11 $\mathrm{n}$ allows you to use cheap and wide available hardware WiFi with MIMO and for previous version experimental verifications theoretical issues provisions of this policy jobs. In the experiment used company's equipment D-Link: wireless router DIR - 6552.4 GHz; wireless the DWA - 547 adapter. Both devices correspond to of the IEEE standard $802.11 \mathrm{n}$ and have a mode MIMO with a mechanism adaptations, similar to that, what is being used in WiMAX. Experimental design scheme installations submitted. To generate traffic and measurements used program level the analyzer IxChariot that was enabled via a subroutine End Point [14-16].

High decorative intensity and duration of flowering in the generic complex Robinia has R. viscosa var. hartwegii (Koehne) Ashe. This species is of great interest for creating solitaire plantings. R. viscosa var. hartwegii is widely recognized in the decorative gardening of arid regions of southern Russia and is used not only in urban gardening objects of General and limited use, but also on private plots as a single ornamental plantWith reduced the graph is visible, what's in the moments map item appearances the analyzer IxChariot programs shows what is noticeable $(>30 \%)$ reducing the number of users capacity abilities when saving values of BER. This indicates that functioning of the mechanism adaptive modulations that saves the specified value probability level bit errors at the expense of reducing the number of users speeds transfers (decrease signalling devices QAM positions). However in $\mathrm{WiFi}$, as in the standard WiMAX, the type of modulation changes in all channels MIMO is the same. One should expect, what is offered adaptive function method V-band modulation individual channels it will be even more so effectively fight against fades out in spatial relations channels [17].

\section{CONCLUSION}

In paper work solved current version scientific and applied task developing methods upgrade transmission quality information in systems radio access WiMAX based on usage adaptive modulations signals in spatial features channels MIMO. The value of the results work for science and practice is developed mathematical models and methods adaptive of modulation, which consider features base stations and terminals users allowed to identify and to offer new practical ways to improve performance fixed mobile WiMAX based on more than full usage spatial, temporary and frequency control resources.

Conclusions and recommendations from the scientific point of view and practical usage received results such:

1. Important direction system development WiMAX with MIMO is their adaptation to change parameters channel to use as completely as possible use all resources time and frequency band regions and minimize it power on the transfer and, at the same time, zoom in pass rate ability communication systems.

2. Development rational ones paths and optimal ones by favorites the criterion algorithms adaptations impossible without objective parameter estimates subchannel b. At the same time, for correct one process analysis physical level necessary apply adequate multipath models channels. In the work for analysis processes in the absence of line of sight used release model, and when joint reception direct and several reflected rays.

3. Measurement parameters channel matrices not completely solves the problem definition real state channel through "freeze" matrices for time packet transfers characters. For mobile devices subscribers channel matrix it may be significant change in time. This leads to towards growth find her definition and, accordingly, to increase bit errors at reception. To increase your sales speeds and accuracy of estimates developed by algorithm $\mathrm{C}$ predictions channel matrices by results previous ones measurements including dynamics of changes these parameters. It is shown that even the simplest one linear prediction channel matrices for MIMO systems with an adaptive feature modulating allows you to additional information reduce the level bit errors in the 
communication system approximately in 3 times.

4. Results modeling they showed that adaptive modulation, provided for standard WiMAX, is not enough effective. Therefore, it is proposed new method is-interactive modulations that got a name "Adaptive V-band modulation MIMO channels". Method it consists of simultaneous when using it different types multi-position modulations in various MIMO channels, depending from attenuation in them. At the strong ones channels used, for example, QAM64, and in weak cases - QAM16 or BPSK. Usage adaptive modulations in MIMO channels provides fixtures communication systems before the change the environment. Winning in SNR can reaching $15 \mathrm{~dB}$ if the amount is insignificant reducing your speed transmitting information. Communication system with adaptation MIMO channels have less than sensitive to speed changes I know that especially important for mobile WiMAX.

5. Displayed possibility and proposed adaptive function method modulations

in frequency band the region. Entity adaptive modulations in frequency band the area is closed

in detection selective ones attenuations that occur in specific frequency band the region. On from this one information changes type of modulation this group subcarriers.

As a result noticeably reduced influence of selective methods fading on the screen communication quality. Received for results the imitation one modeling the SNR win reaches $6 \mathrm{~dB}$.

6. Implementation the adaptive method modulations in MIMO channels provide solutions management tasks. Suggested system model management gear ratio device, in which is used for evaluation channel matrices applied Kalman filter. At the same time, optimization quality of management is being implemented with the use of optimization functions parameters modulations. Selecting a range each of these parameters is being implemented in accordance with a criterion quality based on developed by the algorithm.

7. Simulation modeling conducted using developed software model telecommunications system WiMAX with MIMO and spatio-temporal encoding, which contains replacement software modules environment transmission modulators, demodulators, spatio-temporal the encoders and decoders. Results modeling confirmed main theoretical issues position works and services high efficiency offered methods of adaptation. Results experimental research communication systems they are compatible with MIMO with results theoretical analysis and the imitation one modeling. At the expense of adaptations, even in a difficult situation signal-to-noise ratio environment, real system saves the requested amount transmission quality information.

All the studied species, varieties and forms of the genus Robinia can be used in alley plantings. However, when creating these types of plantings, it is necessary to take into account the propensity of some species to form different life forms depending on the conditions of cultivation (shrub, single-stemmed or multi-stemmed tree) When laying ordinary plantings, in the first years, it is necessary to pay attention to formative pruning to create typical plantings with a similar structure of the system of skeletal branches and trunks. Plants with a strict geometric shape of the crown are of great value for creating alley plantings. In the Robinia genus complex, such plants include R. pseudoacacia f.pyramidalis (Pepin) Rehd. with pyramidal and R. pseudoacacia f. umbraculifera (DC) Rehd. with a spherical crown shape.

The only drawback of these forms is a weak winter hardiness in comparison with other representatives of the generic complex. When designing landscaping objects, it is necessary to select closed areas with a mild microclimate and southern exposure.

In conclusion, it should be noted that all species of the genus Robinia tolerate forming and rejuvenating pruning well, but they are not promising for creating cut forms, since they belong to the group of light-loving plants with an openwork structure of the crown and large complex leaves.

\section{REFERENCES}

1. Peabody F.J. Revision of the genus Robinia (Leguminosae: Papilionoideae). // Ph.D. Dissertation. Ames: Iowa State University, 1984. - 155 p.

2. Isely D., Peabody F. L. Robinia / / Hotel Castanea. 1984. Thom. 49. P. 187-202.

3. Pink-flowered locusts (Robinia L., Fabaceae) established in Poland // Yearbook of the Polish dendrological society, Vol. 63, 2015, p. 9-33

4. Guoqing Li.., Guanghua Xu.., Ke Guo.., Sheng Du Mapping the Global Potential Geographical Distribution of Black Locust (Robinia Pseudoacacia L.) Using Herbarium Data and a Maximum Entropy Model // Forests 2014, №5, p. 2773-2792; doi:10.3390/f5112773

5. Anatoliy Khapugin A global systematic review of publications concerning the invasion biology of four tree species // Hacquetia, №18/2, 2019, p. 233-270 DOI: 10.2478/hacq-2019-0005

6. Vítková, M., Müllerová, J., Sádlo, J., Pergl, J., Pyšek, P. Black locust (Robinia pseudoacacia) beloved and despised: A story of an invasive tree in Central Europe // Forest Ecology and Management, Volume 384, 2017, p. 287-302

7. Bouteiller X. P., Verdu C. F., Aikio E., Bloese P., Dainou K., Delcamp A., De Thier O., Guichoux E., Mengal C., Monty A. A few north Appalachian populations are the source of European black locust // ECOLOGY AND EVOLUTION, volume 9/5, 2019, p. 2398-2414 DOI: $10.1002 /$ ece 3.4776

8. Ortiz P.L. Robinia // Flora iberica. 1999. Vol. 7(1). P. 264-266.

9. The PLANTS Database provides standardized information about the vascular plants, mosses, liverworts, hornworts, and lichens of the U.S. and its territories. URL: https: // plants.sc.egov.usda.gov/java/nameSearch (date of request: 11.09.2019).

10. Vinogradova Yu. K., Tkacheva E. V., Brinzda Ya., Mayorov S. R., Ostrovsky R. To the biology of 
flowering of alien Robinia species / / Russian journal of biological invasions, no. 4, 2012, pp. 10-26

11. Lyudmyla V. Vehera The Use of Robinia L. Genus Representatives in Greenery Optimization of the National Dendrological Park "Sofiyivka" of the National Academy of Sciences of Ukraine // Journal of Native and Alien Plant Studies 15, (2019), p.159-165

12. Lazarev S. E. Features of growth and development of decorative-flowering representatives of the genus Robinia L. Laburnum Medic. and Cercis L. in the dry-steppe conditions of the Volgograd region. // The news of science in agriculture. 2019. No. 1-1 (12). Pp. 86-90.

13. Semenyutina A.V., Lazarev S. E. Diagnostic signs of representatives of the genus Robinia $L$. by generative indicators in introduced populations. // Science. Thought, vol. 9, no. 2, 2019a, pp. 64-94 DOI: 10.25726/worldjournals.pro/WEJ. 2019. 2.4

14. Semenyutina A.V., Lazarev S. E. features of growth and development of representatives of the generic complex Robinia $l$ in the conditions of introduction. // Science.Thought, 20188 (3), from 46-55.

15. Semenyutina A. V., Lazarev E. S., Melnik K. A. evaluation of the reproductive capacity of representatives of ancestral complexes and especially their selection of seed in dry conditions. // Science. Thought, 2019, No. 9 (1), from 46-55.

16. Aida Zamnah, Z. A., Azreena, M. S., \& Saputra, M. B. (2020). C-heart: Augmented reality of 3D heart anatomy. International Journal of Advanced Trends in Computer Science and Engineering, 9(1.1 Special Issue), 430-435.

https://doi.org/10.30534/ijatcse/2020/7191.12020

17. Aini, Q., Hariguna, T., Putra, P. O. H., \& Rahardja, U. (2019). Understanding how gamification influences behaviour in education. International Journal of Advanced Trends in Computer Science and Engineering, 8(1.5 Special Issue), 269-274. https://doi.org/10.30534/ijatcse/2019/4781.52019 